\title{
Uniqueness of Current Cosmic Acceleration
}

\author{
Eric V. Linder \\ Berkeley Lab \& University of California, Berkeley, CA 94720, USA \\ Institute for the Early Universe, Ewha Womans University, Seoul, Korea
}

(Dated: November 1, 2018)

\begin{abstract}
One of the strongest arguments against the cosmological constant as an explanation of the current epoch of accelerated cosmic expansion is the existence of an earlier, dynamical acceleration, i.e. inflation. We examine the likelihood that acceleration is an occasional phenomenon, putting stringent limits on the length of any accelerating epoch between recombination and the recent acceleration; such an epoch must last less than 0.05 e-fold (at $z>2$ ) or the matter power spectrum is modified by more than $20 \%$.
\end{abstract}

\section{INTRODUCTION}

Cosmic acceleration holds the key to physics beyond the standard model of particle physics and gravitation. One of the great puzzles is why its characteristic energy scale is so much less than other energy scales in the standard model. No physical principle seems to explain the finite magnitude required for the cosmological constant. As well, one could ask what is so special about today that acceleration is just now coming to dominate the expansion of the universe. This is even more of a telling argument against a cosmological constant explanation in that its lack of dynamics adds a coincidence problem to the fine tuning. Furthermore, another epoch of accelerated expansion is known in the early universe - inflation and this was decidedly dynamical and not a cosmological constant since it ended.

This leads one to ask: if we have two periods of acceleration, why not more? Could acceleration be an occasional phenomenon? Such a view ameliorates the coincidence problem, since the present is one of many epochs where such physics is manifest. Various models have been proposed to achieve this, e.g. a high energy physics "slinky" potential [1] and stochastic beating between multiple fields [2]. This has also been addressed phenomenologically, notably by [3] , and oscillating field models that can achieve this are common in the literature (e.g. see [4 [6] and references therein). Note that even such a technically natural and well motivated model such as a pseudo-Nambu Goldstone boson [7] in fact goes through numerous cycles of acceleration (see Fig. 3 of [8]). Any scalar field oscillating about a potential minimum will possess equation of state $w=-1$ at the turning points where the kinetic energy is zero, and so can cause acceleration if its energy density is sufficiently large.

So the uniqueness of current acceleration is a question of key interest not just from the perspective of the cosmological constant and the coincidence problem, but also as a guide to the type of physics behind cosmic acceleration. In Sec. III we examine the general characteristics of and constraints on acceleration pre- and post-recombination. In Sec. III we investigate the effects of persistent acceleration post-recombination on the matter density, growth of structure, and distances, placing constraints on the length and onset of any such period for the two general, comprehensive scenarios.

\section{PERSISTENCE OF ACCELERATION}

\section{A. Very Early Universe}

Acceleration in the early universe is difficult to probe, except at particular epochs or if it lasts for many e-folds of expansion. Above $1 \mathrm{TeV}$ in energy, such acceleration falls under the rubric of inflation, and we do not have tight constraints on the energy/time scale or number of individual periods. Once dark matter is detected and understood, we may be able to use the freezeout abundance, involving the competition between the interaction rate and the dilution rate due to expansion, to probe the cosmic expansion in the $1 \mathrm{GeV}-1 \mathrm{TeV}$ region.

Around the time of primordial nucleosynthesis, when the energy scale was $\sim 1 \mathrm{MeV}$, the expansion rate is much better known [9]. Radiation dominates, with total equation of state $w_{\text {tot }}=+1 / 3$. The evolution in the expansion rate, i.e. the Hubble parameter $H$ as a function of scale factor $a$, is less well determined, as seen in Fig. 6 of [9] (also see [10, 11]). Recalling that

$$
\frac{d \ln H^{2}}{d \ln a}=-3\left(1+w_{\text {tot }}\right),
$$

we see that the total equation of state (with $w_{\text {tot }}<-1 / 3$ determining acceleration) is not well known even during this well understood and tested period. We can translate the variation in the slopes of the expansion behaviors in Fig. 6 of [9] into the constraint $-0.4<w_{\text {tot }}<1.4$ for their conservative case and $0<w_{\text {tot }}<1$ for their tighter case.

The recombination and decoupling epochs of the cosmic microwave background (CMB) are also well measured. The decoupling depends again on the competition between the ionization rate and the expansion rate. The explicit dependence on the function $H(a)$ was described analytically by [12], generalizing [13]. Again, the magnitude $H(a)$ is better determined [14] than the logarithmic derivative $d \ln H^{2} / d \ln a$ that gives information on the total equation of state. Acceleration during and pre-recombination will be treated in future work. 


\section{B. Post-Recombination Acceleration}

We concentrate on possibilities for acceleration between decoupling and the onset of the current acceleration, at redshifts $z \approx 1-1000$. Most of this period is not well probed by distance measures (but see the next section) so we do not have direct measures of the expansion. However, acceleration breaks matter domination, diluting the matter density and affecting the growth of structure in two ways. First, it reduces the source term for matter perturbation growth, and second it increases the Hubble friction term, proportional to $3-q$ where $q \equiv-a \ddot{a} / \dot{a}^{2}$ is the deceleration parameter. In terms of the total equation of state, the friction term is proportional to $5-3 w_{\text {tot. }}$ See Eq. (8) of [15] and Sec. 4.1 of [16] for more details.

The effects of intermediate acceleration on growth were discussed for certain models in detail by [17]. That article found two key effects on growth: the suppression during the actual period of acceleration, but also the stunting of the growth rate, i.e. the velocity $\dot{\delta}$ of the perturbations, that persisted to much later times. We continue the exploration here, allowing more degrees of freedom to the model for acceleration and looking for analytic scalings of the effects, while giving a more general and systematic treatment.

Consider the total equation of state of the universe,

$$
w_{\text {tot }}=w \Omega_{w}(a)
$$

for a flat universe. To attain acceleration a necessary but not sufficient condition is $w<-1 / 3$; one requires $w_{\text {tot }}<-1 / 3$. Equation (2) immediately suggests two alternatives for achieving $w_{\text {tot }}<-1 / 3$. The first is to have a large dark energy fraction $\Omega_{w}(a)$, hence dark energy will dominate over matter. However, once the dark energy dominates it will continue to do so while $w<0$ and we do not achieve the desired occasional, or stochastic, nature of the accelerating epoch. Thus in this approach we will need to allow $w>0$ at some time to undo the dark energy domination. We refer to this as the "superdecelerating" scenario.

The second approach is to keep $\Omega_{w}(a)<\Omega_{m}(a)$ but attain acceleration through a strongly negative dark energy equation of state. Thus the universe accelerates but dark energy does not dominate - this is in fact exactly what happens in $\Lambda \mathrm{CDM}$ in the range $z \approx 0.4-0.7$. Then there is no need for a subsequent period that reduces the dark energy density fraction, hence no need for $w>0$. We will see that in order to get an early acceleration period separate from the current acceleration, this requires $w \ll-1$. We therefore refer to this alternative as the "superaccelerating scenario".

Figures 1,2 illustrate the characteristics of the two different scenarios. Note that the superacceleration case has a lower value of the dark energy density in the past than today (effectively a smaller cosmological constant), while the superdeceleration case has a higher value. We show the behaviors of the matter density $\rho_{m}(a)$, dark energy density $\rho_{w}(a)$, and the Hubble parameter $H^{2}(a)$, as well as the dark energy equation of state $w(a)$. For the last quantity we take a simple model of a constant deviation down (up) from the cosmological constant value $w=-1$ for superacceleration (superdeceleration), for $N$ e-folds of expansion. Note that the number of e-folds of early acceleration, $N_{\text {acc }}$ is much smaller than the duration $N$ of the deviation in $w$. The periods of acceleration are shown by the thick parts of the $\rho_{w}$ or $w$ curves.

\section{PATHS TO ACCELERATION}

Let us examine the scenarios in detail. These two possibilities of superacceleration and superdeceleration span the range of ways to achieve acceleration, stemming from the simple definition $w_{\text {tot }}<-1 / 3$.

\section{A. Superaccelerating Scenario}

In the superacceleration scenario one has a down-thenup transition in the dark energy equation of state. We model this as a simple step away from a constant $w$, to a constant $w_{j}$, lasting for a number of e-folds $N=\Delta \ln a$ and then returning to $w$ at scale factor $a_{u}=1 /\left(1+z_{u}\right)$ (so the overall deviation begins at $a_{d}=a_{u} e^{-N}$ ). We usually take $w=-1$ so that before and after the deviation the universe follows $\Lambda \mathrm{CDM}$; in any case it is matter dominated at high redshift and has a present epoch of acceleration. All physical quantities such as densities are continuous, while if we had made steps in $\rho$ or $H$ then we would have faced infinities in the equation of state. Although there is no problem with our sharp steps, one could also use a smoothed form for $w(a)$ capable of rapid transitions, such as the e-fold model [18]. Such formal smoothing makes no difference to the results.

The dark energy density never dominates until the usual time near the present, but nevertheless a highly negative equation of state can drive accelerated expansion. The condition for accelerated expansion is determined by the total equation of state:

$$
\begin{aligned}
w_{\text {tot }} & =w \Omega_{w}\left(a_{d}<a<a_{u}\right)=w_{j}\left[1+\frac{\Omega_{m}}{\Omega_{w}} a_{u}^{-3\left(w_{j}-w\right)} a^{3 w_{j}}\right]^{-1} \\
& <-\frac{1}{3}
\end{aligned}
$$

This imposes the requirement that to obtain any acceleration one needs

$$
w_{j}<-\frac{1}{3}\left(1+\frac{\Omega_{m}}{\Omega_{w}} a_{u}^{3 w}\right) .
$$

If we want the early acceleration to be distinct from the current acceleration, then we could impose $z_{u}>2$, say, implying $w_{j}<-3.8$ (taking $\Omega_{m}=0.28$ ). Thus we indeed require a highly negative equation of state. 

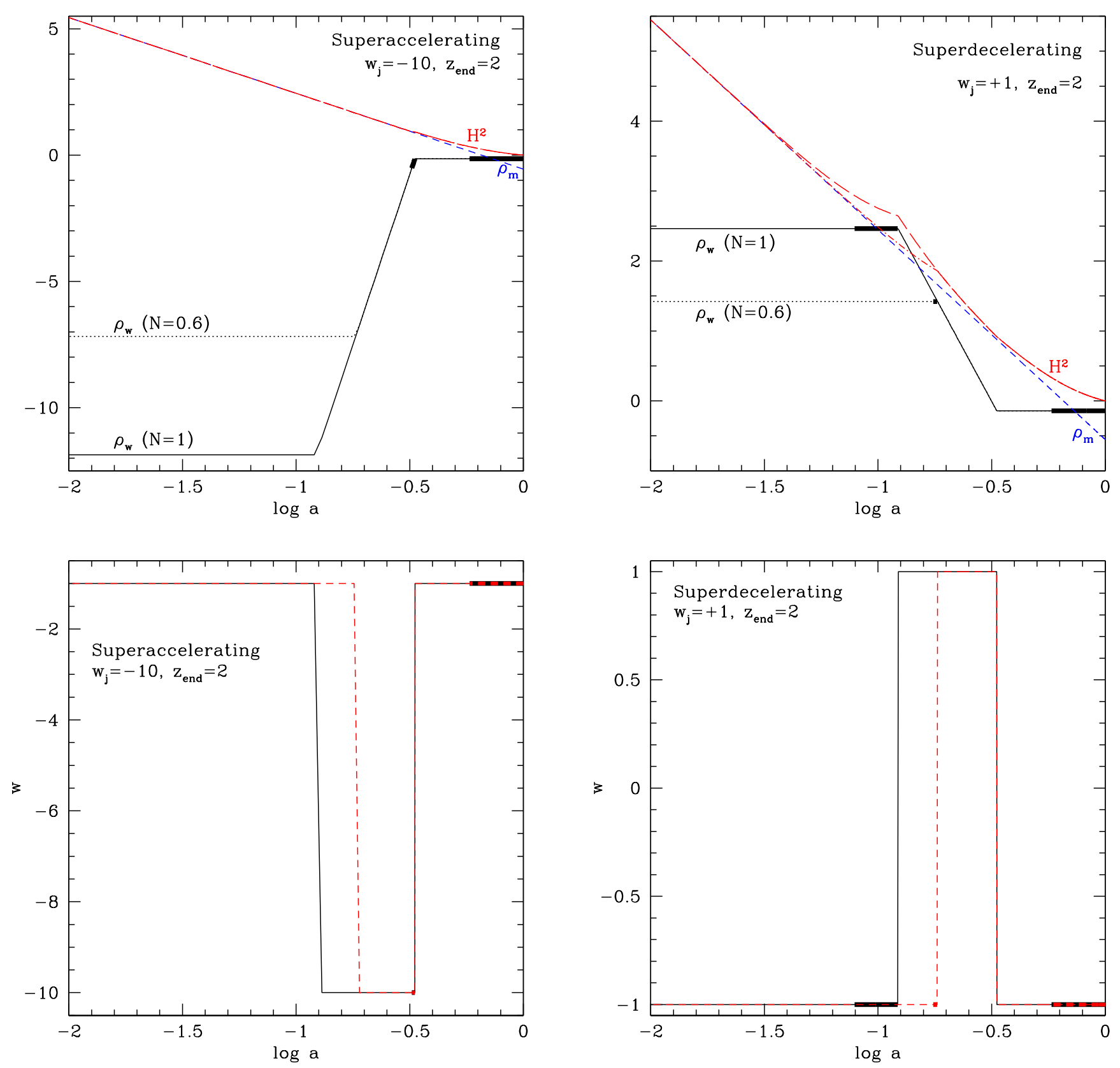

FIG. 1: The superaccelerating scenario has a period of strongly negative dark energy equation of state $w_{j}<-1$, which drives $w_{\text {tot }}<-1 / 3$ and so causes acceleration. The top panel plots the logs of the dark energy density $8 \pi G \rho_{w}(a) /\left(3 H_{0}^{2}\right)$, dark matter density $8 \pi G \rho_{m}(a) /\left(3 H_{0}^{2}\right)$, and Hubble parameter $H^{2} / H_{0}^{2}$ vs. the log of the scale factor $a$. The $w_{j}<-1$ epoch is taken to last for $N=0.6$ or 1 e-folds, ending at $z_{u}=2$. However, the actual acceleration lasts for a much shorter time, shown by the thick portions of the $\rho_{w}$ curves (including for the current epoch of acceleration). In particular, note the early acceleration only lasts for 0.034 efolds. The bottom panel plots $w(a)$ on the same horizontal scale so one can see directly the effect of $w(a)$ on the densities. The accelerated epochs are again indicated by the thick parts of the curves.

FIG. 2: As Fig. 1 but for the superdecelerating scenario. Here the acceleration occurs because of relatively large $\rho_{w}(a)$, rather than supernegative $w$. To bring the dark energy density to the current level then requires a period of superdeceleration, where $w>0$ to redshift away the excess density. Note that in the $N=0.6$ case the dark energy never dominates the matter density during the early acceleration.

In general the number of e-folds of accelerating expansion is given by

$$
N_{\mathrm{acc}}=\frac{1}{-3 w_{j}} \ln \left[\frac{\Omega_{w}}{\Omega_{m}} a_{u}^{-3 w}\left(-3 w_{j}-1\right)\right] .
$$




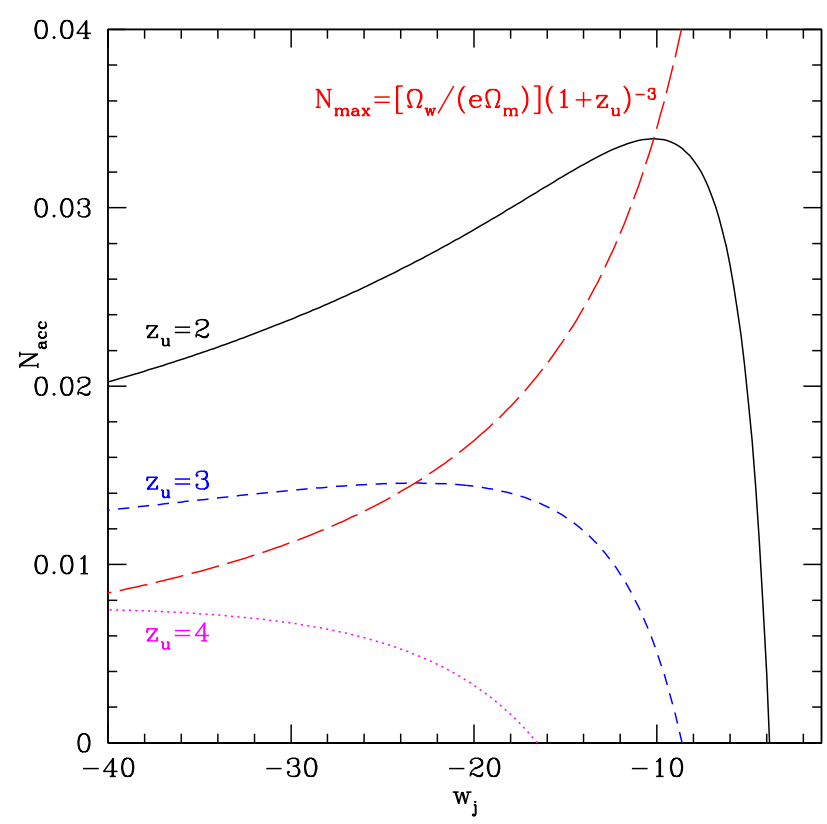

FIG. 3: The superaccelerating scenario can only support a very small number of e-folds of acceleration because for a supernegative equation of state $w_{j}$ the dark energy density must start very small to end up at the observed level today. The plot shows the number of e-folds of acceleration as a function of $w_{j}$ and the redshift $z_{u}$ when superacceleration ends. The red, long-dashed curve show the loci of the maximum number of e-folds for each choice of $z_{u}$ and its equivalent $w_{j}\left(N_{\max }\right)$. For an early period of acceleration sufficiently distinct from the present acceleration, e.g. $z_{u}<2$, less than 0.034 e-folds of acceleration are allowed.

This is plotted in Fig. 3 as a function of $w_{j}$ for various values of $z_{u}$. (Note that it is actually independent of the number of e-folds the $w_{j}$ transition lasts, as long as $N>N_{\text {acc. }}$.) The maximum number of e-folds allowed for a given $z_{u}$ is

$$
N_{\mathrm{acc}, \max }=\frac{1}{-3 w_{j, \max }-1} \approx \frac{\Omega_{w}}{e \Omega_{m}} a_{u}^{-3 w},
$$

where $w_{j, \max }$ is the value of $w_{j}$ that maximizes Eq. (5) for a fixed $a_{u}$. For example, for $z_{u}=2$ and $w=-1$, one has $w_{j, \max }=-10.3$ and $N_{\mathrm{acc}, \max }=0.034$. This relation is shown in Fig. 3 as the red, long-dashed curve.

Increasing the redshift of the early acceleration, to alleviate the coincidence problem more convincingly, decreases the period of acceleration allowed. Because such a highly negative equation of state is required, this means that the dark energy density grows rapidly, $\rho_{w} \sim a^{-3\left(1+w_{j}\right)}$, so to achieve the same $\Omega_{w}$ today requires a small $\rho_{w}$ at early times. Moving the acceleration period to earlier times reduces $\Omega_{w}(a)$ for the same $\rho_{w}(a)$, and this in turn makes it harder to achieve $w_{\text {tot }}=w \Omega_{w}(a)<-1 / 3$ at early times. Attempting to drive $w_{j}$ even more negative simply diminishes early
$\Omega_{w}(a)$ even more, and so this scenario can never achieve long periods of early acceleration.

In summary, at best far less than a single e-fold, only $N \approx 0.03$, of early acceleration can be achieved. Thus one seems to replace a coincidence problem with a further fine tuning issue: why should acceleration last for such an apparently unnaturally short time, much less than one e-fold? Inflation lasted for some 60 e-folds and current acceleration appears to have existed for at least half an e-fold so far.

Since the number of early acceleration e-folds is so small, it should have negligible effect on growth or distance probes. For example, even $N=1$ e-fold of $w_{j}=-10$, ending at $z_{u}=2$, changes the total growth to the present by only $1.7 \%$ relative to the standard $\Lambda \mathrm{CDM}$ case. Oddly, growth is actually increased by such an acceleration scenario since the diminished dark energy density for much of the history slightly increases $\Omega_{m}(a)$.

The velocity effect on the growth, i.e. the stopping and restarting of the growth due to acceleration and restoration to deceleration, can be measured via the LinderWhite 19] matching prescription for the nonlinear matter power spectrum (cf. [17]). Here, the key parameter is the growth ratio $R_{g}=g(a=0.35) / g_{0}$ where $g(a)=D(a) / a, g_{0} \equiv g(a=1)$, and $D(a) \sim \delta(a)$ is the matter perturbation growth factor; this is only suppressed by $0.1 \%$, due to the extremely short duration of the acceleration. The distance to CMB last scattering is increased by $0.4 \%$, essentially totally from the actual acceleration period, not the transition to $w_{j}$ per se.

\section{B. Superdecelerating Scenario}

If, unlike in the superaccelerating scenario, the dark energy density dominates during the early acceleration, then this must be undone to restore matter domination allowing growth of structure. That is, there must be an epoch after acceleration in which the dark energy redshifts away more swiftly than the matter so the matter can rise (again) to dominance. This requires a period when $w>0$, which we call superdeceleration.

In order to have early acceleration, one will need to have a higher than standard dark energy density at high redshift, in order to obtain the same dark energy density today despite the extra dilution (see Fig. 2). In fact, the dark energy does not even have to dominate, only have $\Omega_{w}(a)>1 /(-3 w)$, for example $\Omega_{w}(a)>1 / 3$ for $w=$ -1 , to give rise to acceleration. We take a model with $\Lambda \mathrm{CDM}$ at high redshift, though with extra dark energy density. This high value of dark energy density kicks off a period of early acceleration, lasting for $N_{\text {acc }}$ e-folds. Then, at $z_{j}$ the dark energy equation of state jumps up to $w_{j}>0$ (note the jump is up not down, in contrast to the superacceleration scenario). This superdecelerating phase lasts for $N_{\text {dec }}$ e-folds, then $w=-1$ is restored and the expansion proceeds again as $\Lambda \mathrm{CDM}$.

Figure 4 shows the behavior of the fractional dark 


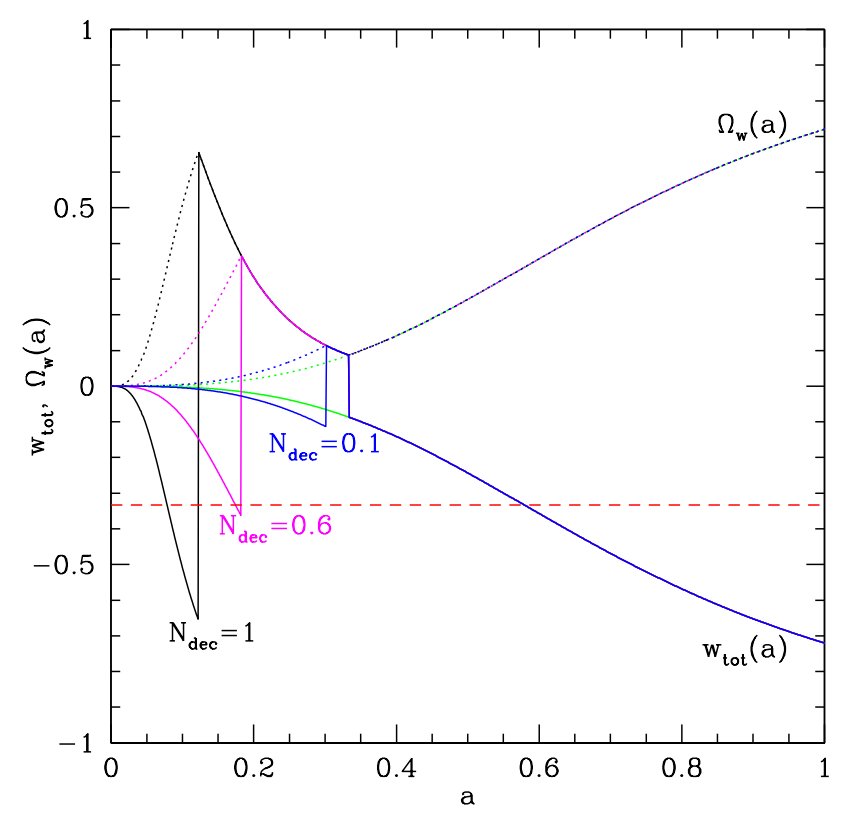

FIG. 4: The fractional dark energy density $\Omega_{w}(a)$ (dotted, positive only curves) and total equation of state $w_{\text {tot }}(a)$ (solid curves) are plotted for the superdecelerating scenario with three different values of $N_{\mathrm{dec}}$. Note that the $N_{\mathrm{dec}}=0.1$ case never achieves early acceleration $\left(w_{\text {tot }}<-1 / 3\right.$, shown by the horizontal dashed line), the $N_{\mathrm{dec}}=0.6$ case barely does, and even the $N_{\mathrm{dec}}=1$ model has only a short period of early acceleration. All models shown have $w_{j}=+1$ and return at $z=2$ to $\Lambda$ CDM with $\Omega_{w}(a=1)=0.72$. The smooth, green curves correspond to $\Lambda$ CDM without any transition.

energy density $\Omega_{w}(a)$ and total equation of state $w_{\text {tot }}$. The recent universe is standard $\Lambda \mathrm{CDM}$, while there are epochs of acceleration $\left(w_{\text {tot }}<-1 / 3\right)$ and superdeceleration $\left(w_{\text {tot }}>0\right)$ in the early universe. The dark energy density is boosted at high redshift relative to the standard case.

One sees that a substantial period of superdeceleration is required to permit even a brief period of early acceleration. Indeed, no early acceleration can occur unless

$$
N_{\mathrm{dec}}>\frac{1}{3\left(1+w_{j}\right)} \ln \left[\frac{\Omega_{m}}{2 \Omega_{w} a_{j}^{3}}\right] .
$$

For $z_{j}=4$, for example, one requires $N_{\text {dec }}>0.53$. In general, the number of e-folds of early acceleration, $N_{\text {acc }}$, is related to $N_{\mathrm{dec}}$ and the transition scale factor from acceleration to deceleration, $a_{j}$, by

$$
N_{\mathrm{acc}}=N_{\mathrm{dec}}\left(1-\frac{w_{j}}{w}\right)+\ln a_{j}-\frac{1}{3 w} \ln \frac{2 \Omega_{w}}{\Omega_{m}},
$$

where $w$ is the dark energy equation of state outside the superdeceleration period (i.e. $w=-1$ if we take $\Lambda \mathrm{CDM}$ as the baseline).

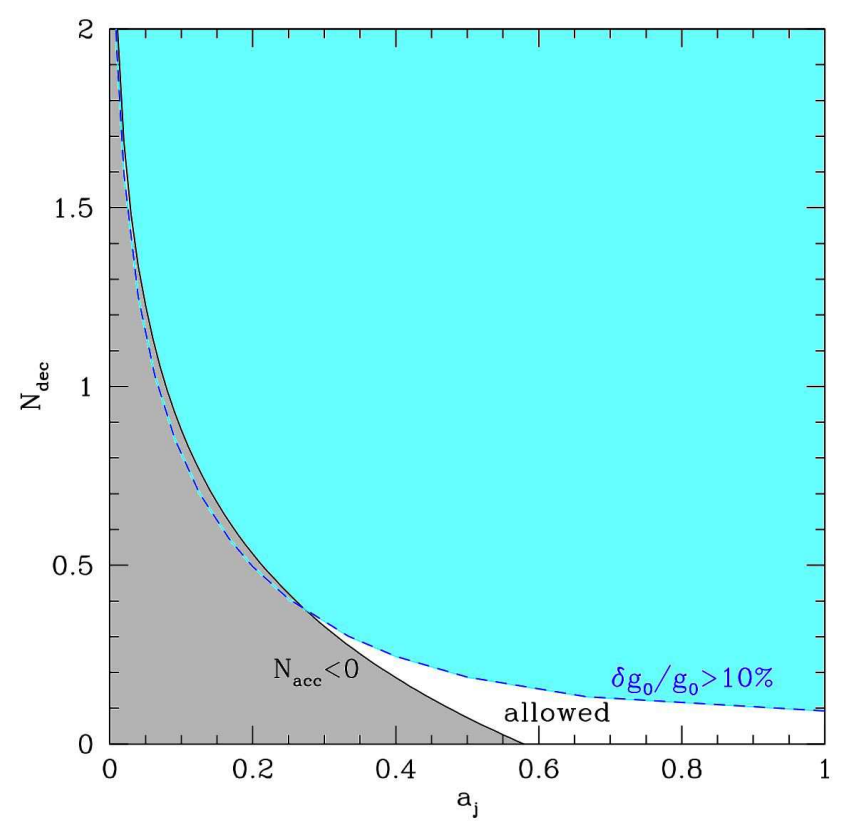

FIG. 5: Early acceleration due to a high early dark energy density can only occur, and still deliver the same dark energy density today, if it is followed by sufficient superdeceleration (here $w_{j}=+1$ ). Only the space above the solid, black curve, giving $N_{\text {acc }}>0$, achieves this. Too much superdeceleration, however, strongly suppresses the total growth to the present. Only the space below the dashed, blue curve diminishes the growth relative to $\Lambda$ CDM by less than $10 \%$. The only surviving region is the thin crescent between the curves at $a_{j}>0.27$, or $z_{j}<2.7$, which hardly qualifies as early acceleration.

Equation (7) gives a lower limit on $N_{\mathrm{dec}}$ in terms of $a_{j}$. If $N_{\mathrm{dec}}$ is too large, however, then growth will be severely affected. Recall that any deviation from matter domination, whether acceleration or superdeceleration, can suppress growth. This constraint then gives an upper limit on $N_{\text {dec }}$ so we can evaluate the viability of the superdecelerating scenario for early acceleration based on the comparison of these upper and lower limits. From Fig. 5 we see that in fact superdeceleration fails.

Only a thin sliver of parameter space for $z_{j}<2.7$ survives, and such a late epoch of acceleration does nothing to help the coincidence problem (decreasing $w_{j}<+1$ worsens the situation). In addition, the length of the acceleration is quite short: for $z_{j}=1.5$, say, one has at best a number of e-folds $N_{\text {acc }}=0.12$. This does not seem like a very natural value, being much less than an e-fold. Attempting to create several epochs of acceleration, necessitating even shorter durations, simply exacerbates the problems. At high redshift, the curve $N_{\text {acc }}=0$ roughly corresponds to $\delta g_{0} / g_{0}=15 \%$ (one can think of this roughly as a shift in the mass amplitude $\sigma_{8}$ ), or $30 \%$ deviation in the matter power spectrum amplitude.

The growth velocity factor, measured through $R_{g}$, is affected at the $5.1 \%(2.7 \%)$ level for $z_{j}=1.5(2.0)$ and the 
maximum number of e-folds of acceleration, 0.12 and 0.05 respectively. More severe is the impact on the distance to last scattering, mostly due to the higher early dark energy density: this is reduced by $2.9 \%$ (2.7\%), which essentially means that even the thin sliver of allowed parameter space in Fig. 5 is in doubt when including CMB constraints. Compensating by decreasing the matter density can preserve the distance to last scattering, but worsens the growth deviation.

\section{CONCLUSIONS}

Only two paths exist to obtaining a period of cosmic acceleration: the dark energy density is subdominant but its equation of state is highly negative (superacceleration), or the dark energy density is dominant, or nearly so, and its equation of state is at least moderately negative (superdeceleration). In the first case, we have seen that the dynamics unavoidably forces the dark energy density to be so low that acceleration is quite transient - less than 0.035 e-folds for acceleration before $z=2$. This conclusion only depends on a dark energy density fraction today $\Omega_{w} \approx 0.7$, not on any external data.

In the second case, the acceleration is caused by an unusually high early dark energy density, which then must be diluted in order to restore matter domination. To accomplish this, the dark energy equation of state must become positive, hence leading to a period of superdeceleration. A period of acceleration requires a longer period of superdeceleration, yet these both suppress the growth of matter density perturbations. The two constraints of requiring sufficient superdeceleration to allow for some early acceleration, yet not disrupting growth, pinch the allowed parameter space to permit at most 0.05 e-folds of acceleration before $z=2$.

Thus, neither possibility for achieving $w_{\text {tot }}<-1 / 3$ - via superacceleration or superdeceleration - can truly provide early, or occasional acceleration, or provide any help in ameliorating the coincidence problem. It is interesting that such a clear conclusion falls out from such simple arguments. The current acceleration indeed appears to be unique since the time of $\mathrm{CMB}$ decoupling.

\section{Acknowledgments}

I thank Stephen Appleby, Marina Cortês, Roland de Putter, Manoj Kaplinghat, and especially Tristan Smith for useful discussions. This work has been supported in part by the Director, Office of Science, Office of High Energy Physics, of the U.S. Department of Energy under Contract No. DE-AC02-05CH11231, and the World Class University grant R32-2009-000-10130-0 through the National Research Foundation, Ministry of Education, Science and Technology of Korea.
[1] G. Barenboim, O. Mena, C. Quigg, JCAP 0604, 008 (2006) arXiv:astro-ph/0510178

[2] S. Dodelson, M. Kaplinghat, E. Stewart, Phys. Rev. Lett. 85, 5276 (2000) arXiv:astro-ph/0002360

[3] K. Griest, Phys. Rev. D 66, $123501 \quad$ (2002) arXiv:astro-ph/0202052

[4] G. Mangano, G. Miele, V. Pettorino, Mod. Phys. Lett. A 18, 831 (2003) arXiv:astro-ph/0212518

[5] B. Feng, M. Li, Y-S. Piao, X. Zhang, Phys. Lett. B 634, 101 (2006) arXiv:astro-ph/0407432

[6] E.V. Linder, Astropart. Phys. 25, 167 (2005) arXiv:astro-ph/0511415

[7] J. Frieman, C. Hill, A. Stebbins, I. Waga, Phys. Rev. Lett. 75, 2077 (1995) arXiv:astro-ph/9505060

[8] R. de Putter \& E.V. Linder, JCAP 0810, 042 (2008) arXiv:0808.0189.

[9] S.M. Carroll \& M. Kaplinghat, Phys. Rev. D 65, 063507 (2002) arXiv:astro-ph/0108002

[10] E. Masso \& F. Rota, Phys. Rev. D 68, 123504 (2003)
arXiv:astro-ph/0302554

[11] S. Dutta \& R. Scherrer, arXiv:1006.4166

[12] E.V. Linder, arXiv:astro-ph/9712159

[13] B.J.T. Wyse \& R.F.G. Jones, Astr. Astrophys. 149, 144 (1985)

[14] S. Galli, A. Melchiorri, G.F. Smoot, O. Zahn, Phys. Rev. D 80, 023508 (2009) arXiv:0905.1808.

[15] E.V. Linder, Phys. Rev. D 72, 043529 (2005) arXiv:astro-ph/0507263

[16] E.V. Linder, Rept. Prog. Phys. 71, 056901 (2008) arXiv:0801.2968

[17] E.V. Linder, Astropart. Phys. 26, 16 (2006) arXiv:astro-ph/0603584

[18] E.V. Linder \& D. Huterer, Phys. Rev. D 72, 043509 (2005) arXiv:astro-ph/0505330

[19] E.V. Linder \& M. White, Phys. Rev. D 72, 061304 (2005) arXiv:astro-ph/0508401 